\title{
Deployment of the WIRELESS SENSOR NETWORK (WSN) using a Computer Vision based AGV - (Mobile Robots)
}

\author{
Rajiv A ${ }^{1 *}$ Lakshmi C ${ }^{1}$ Divya $\mathrm{M}^{2}$ \\ ${ }^{1}$ Assistant Professor, ECE Sriram Engineering College, Perumalpattu -602 024 \\ ${ }^{2}$ Research Associate, Vellore Institute of Technology, Chennai \\ rajiva.ece@sriramec.edu.in,lakshmic.ece@sriramec.edu.in,divyamanne16@gmail.com
}

\section{ABSTRACT}

The objective of this paper is to deploy the Wireless Sensor Network (WSN) using a Computer Vision Based AGVs (Automated Guided vehicle-Robot) and Make Sensor Networks Practical with Robots for Post Disaster Management. Beyond disaster Management these Robots can also be used as the substitute for Solider in the future war fare to reduce loss of human life as far as possible. In our proposed system, Mobile Robots carry 4 types of sensors (PIR sensor, Metal Detector, Haptic robotic Arm \& fire, temperature sensor) for human detection \& also for rescue.

"why to put human lives at risk when robots can do their job much more effectively?"

This simple question is answered by our paper.

It is very essential to have a robot during disaster conditions like Earthquake or Bomb blast, where we have to identify live human beings as quickly as possible to save life. Our ultimate Aim was to have two mobile Robots. $1^{\text {st }}$ is Surveillance Robot (here after Target Robot) and $2^{\text {nd }}$ is Rescuer Robot The Communication between the Target Robot and the Rescuer robot is Made by 802.15.4 and the base station communication can be made by Standard Wireless Network of IEEE $802.11 \mathrm{~g}$. The Target robot can easily navigate through rough terrain without getting stuck since it can detect obstacles and also it Detect fire, poisonous gases, Enemy remote vehicles. The Rescuer robot uses following methods to detect a human, one is using the IR radiation emerging from the live humans and contactless sensor MIR (microwave impulse radar) for detecting the heart beat of Human . Our has an robotized arm with six degrees of freedom so that robots can diffuse bomb and unexploded devices, thus saving lives and limbs of many soldiers Provided The robot has a scissor Lift for Lifting heavier debris or Metal Rods and rescue the human.

KEY WORDS: 802.11 g communication, microwave impulse radar 1 : G* Target Robot ,2: Rescuer robot, 802.15 .4 (long battery life $\&$ effective communication for inter robot communication)
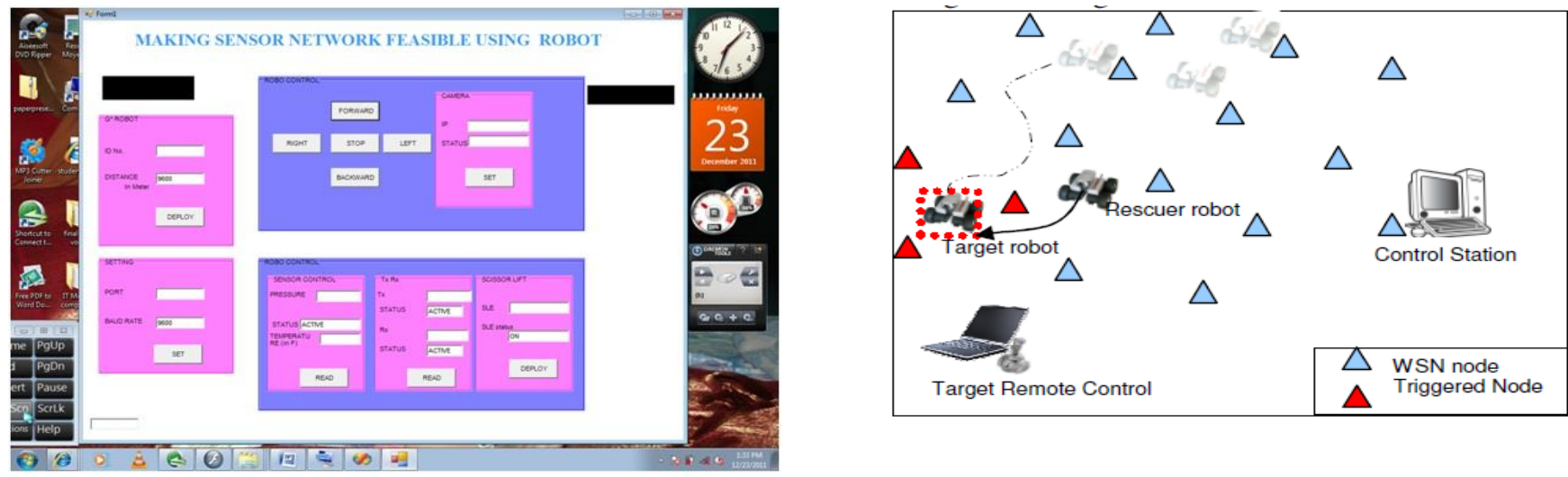

www.trendytechjournals.com 


\section{Introduction}

\section{A. About the Paper}

The main aim of this project is to design a robot which will be controlled through the pc which is located in the remote location and the data will be transmitted to the robot through wireless $802.11 \mathrm{~g}$ standard wireless communication network. The special feature of the robot is human voice reorganization. We are going to design a robot, which will be controlled through pc from any remote location and the robot movements like forward, reverse, left, right can be controlled from the remote location through the pc keypad. The robot model will be connected to the micro controller kit and operated independently. The robot will have the following sensors like intruder sensor; fire sensor, temperature sensor and the set values will be given. It will constantly monitor the location while the movements can be controlled from the remote location through wireless RF transmitter and if the anything occurs like fire, the data will be transmitted from the robot to the pc. Similarly if the temperature set value exceeds the limit, data will be transmitted to the pc and the alarm will be given. In the robot we are going to have a receiver to receive the commands from the pc, sensors like fire and temperature to monitor the conditions, intruder sensor to sense any obstacle and take deviation, wireless RF transmitter to transmit the above data.

\section{B. Need for The Project}

Generally, Rescue People cannot enter into some parts / places of the war field or in the earth quake affected areas due to the situations aroused.

But actually speaking

1) Some people may be alive in that field(s)

2) Some people may be in the unconscious status

3) Some people may be injured / affected badly and cannot move around

To overcome this, or to avoid such unwanted situations, we are going to develop an Alive Human Being Detector using PIR sensor and live working model

\section{Applications}

Remote Keyless Entry (RKE)

Remote Lighting Controls

On-Site Paging

Asset Tracking

Wireless Alarm and Security Systems

Long Range RFID

Automated Resource Management

\section{Advantages}

Ours is a Wireless ROBOT with Wireless Movements 360 degree rotation

Ours also has a special and main function of sensing Infrared waves emitting from the human bodies. voice recognition with multisensoryllI

\section{Proposed System}

We are going to design a robot, which will be controlled through pc from any remote location and the robot movements like forward, reverse, left, right can be controlled from the remote location through the pc keypad. The robot model will be connected to the micro controller kit and operated independently. The robot will have the following sensors like intruder sensor; fire sensor, temperature sensor and the set values will be given. It will constantly monitor the location while the movements can be controlled from the remote location through wireless RF transmitter and if the anything occurs like fire, the data will be transmitted from the robot to the pc.

\section{Design and Implementation}

A. Description In this paper, we will have the Robot model, which will roam around the war field / Earth quake affected area, which has a free forward / Reverse and full 360 degree movement, to move into the war filed / earth quake affected area, to find out the Alive or Survived Human being(s). The Robot will have the PIR sensor,\& IR camera which will help to detect the alive human beings, it consists of an IR based human radiation sensor, which picks up signals from human body radiations and gives a signal output. The human body radiates infrared waves with wavelengths of 8 to 12 micrometers. Whenever any human being comes in the vicinity of the system (up to $1 \mathrm{~m}$ ) the IR system gives the Signal. The Robot also has a wireless Transmitter IEEE 802.11g and sends the message to the Remote Location whenever it finds any alive human. And also insist the 
Rescuer robot through IEEE 802.15.4(Low Rate WPAN) deals with low data rate but very long battery life (months or even years) and very low complexity.

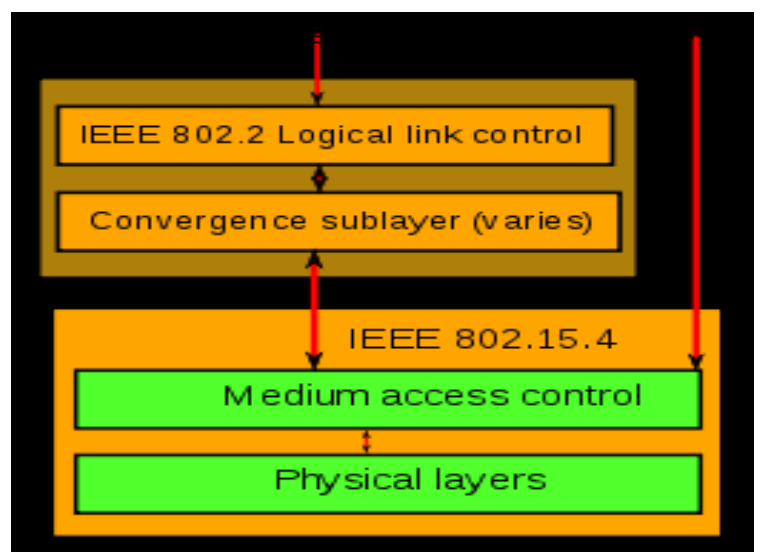

Specialization of 802.15.4 The standard defines both the physical (Layer 1) and data-link (Layer 2) layers of the OSI model. The first edition of the 802.15.4 standard was released in May 2008 several standardized and proprietary networks (or mesh) layer protocols run over 802.15.4-based networks, including ieee 802.15.5, zigbee, 6lowpan, wireless hart, and isa100.11a

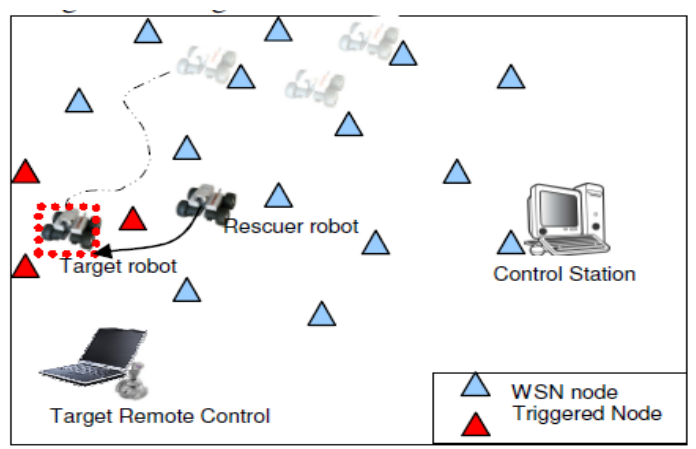

B. Block Diagram

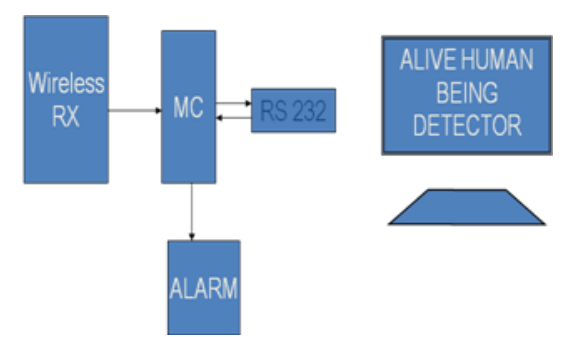

Fig. 1.Target Robot

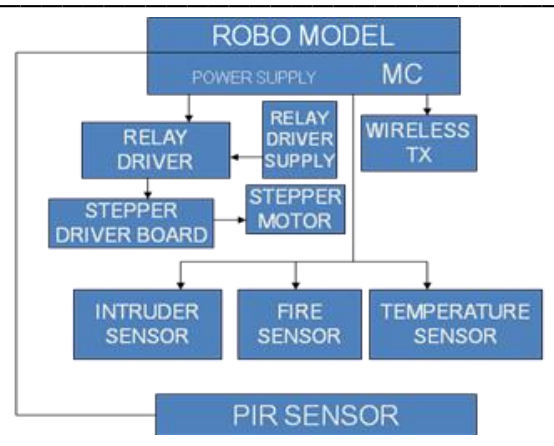

Fig. 2 Rescuer Robot

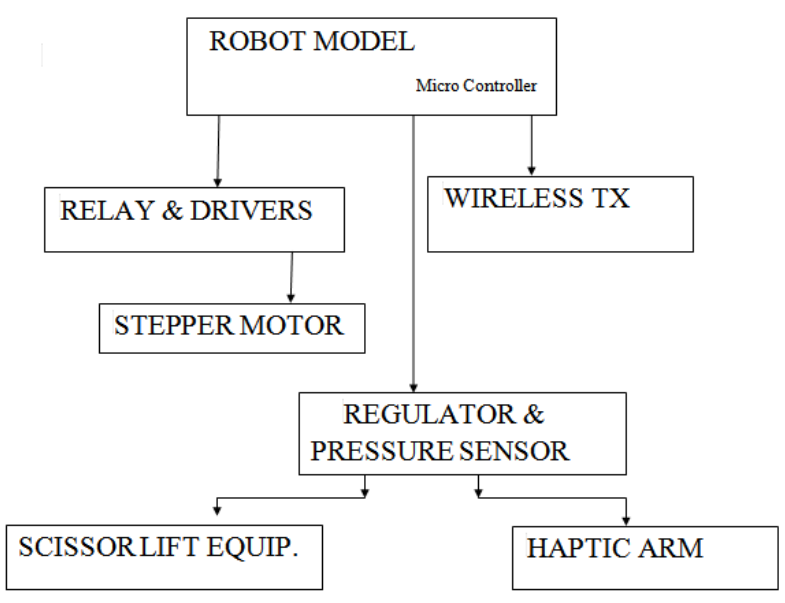

\section{Microcontroller Board}

This is the board, which contains Micro controller Chip as well as the clock Circuitry, Reset Circuit and Power Supply for the Micro controller. A micro controller is a highly integrated chip, which includes, on one chip, all or most of the parts needed for a controller. The micro controller could be called a "ONE-CHIP SOLUTION".It typically includes: CPU (central processing unit) RAM (Random Access Memory) EPROM/PROM/ROM (Erasable Programmable Read Only Memory) I/O (input/output) - serial and parallel timers interrupt controller By only including the features specific to the task (control), cost is relatively low.

A typical micro controller has bit manipulation instructions, easy and direct access to I/O (input/output), and quick and efficient interrupt processing. Micro controllers are a "one-chip solution', which drastically reduces parts count, and design costs 


\section{Control Circuit}

This Board is used to convert the commands given by the Cell Phone / Remote Phone / Remote Control, to logic level signal and provides the input to the embedded micro controller

\section{Flame Sensor}

LDR's are employed to sense the Fire, Here we use powerful and sensitive "LDR" (Light Dependent Resistor) for the flame detection. Normally LDR senses all the lights. But in our case we have to sense only Blue and Yellow flame and reject sunlight and other luminaries. We have provided necessary circuit to reject ambient and luminaries lightings and senses only desired flame coloring. We are using simple and effective potential divider to find out flame.Temperate Indicator \& Controller In certain industries we have to maintain the temperature below $20 \varnothing \mathrm{C}$ to safe guard the electronic from thermal runaway failures. We are using thermistor (THERMAL RESISTOR), which comes under passive transducer classification. Thermistor finds wide applications and advantages like Fast response, smaller in size, Rugged (Not affected by shock and vibration), Good sensitivity, Low cost. A thermistor is a ceramic semiconductor, which exhibits a large change in resistance with a change in its body temperature. The word thermistor is actually a contraction of the words "THERMAL RESISTOR".

\section{Intruder Detection \& Alarm System}

The systems, which detect intruder, are known as intruder detection system. Photoelectric sensors and detection systems use a light-sensitive cell and a directed light source, which points, directly at the cell. The source transmits invisible light beams to the adjacent sensor. Often an infrared filter covers the light source to make it non-visible to intruders If an intruder crosses the light beam, it breaks the beam's continuous contact with the photoelectric Sensor and trips the alarm.

PIR sensor

- $\quad$ Detect the alive human beings

- Consists of an IR based human radiation sensor

- Human body radiates IR of 8 to $12 \mu \mathrm{m}$ Wave length

- $\quad$ Senses up to $1 \mathrm{~m}$

\section{Thermal imaging:}

Our eyes are detectors that are designed to detect visible light (or visible radiation). There are however other forms of light (or radiation) that we can not see. The human eye can only see a very small part of the electromagnetic spectrum. At one end of the spectrum we can not see ultraviolet light, while at the other en our eyes can not see infrared. Infrared lies between the visible and microwave portions of the electromagnetic spectrum.

- The primary source of infrared radiation is heat or thermal radiation. Any object that has a temperature above absolute zero (-273.15 degrees Celcius or 0 degrees Kelvin) emits radiation in the infrared region. Even objects that we think of as being very cold, such as ice cubes, emit infrared radiation. The heat that we feel from sunlight, a fire or a radiator is all infrared. Although our eyes can not see it, our skin can feel it as heat. The warmer the object, the more infrared radiation it emits.

- How does a thermal imaging camera work? Infrared energy coming from an object is focused by the optics onto an infrared detector. The detector sends the information to sensor electronics that translates the data into an image that can be viewed on any standard LCD. Based on the temperature differences of objects, a thermal imaging camera can produce a comprehensive image on which the smallest of temperature differences can be seen.

- Contrary to other technologies, such as e.g. light amplification, that need at least small amounts of light to generate an image, thermal imaging needs no lights at all 


\section{International Journal of Trendy Research in Engineering and Technology \\ Volume 5 Issue 5 October 2021 \\ ISSN NO 2582-0958}

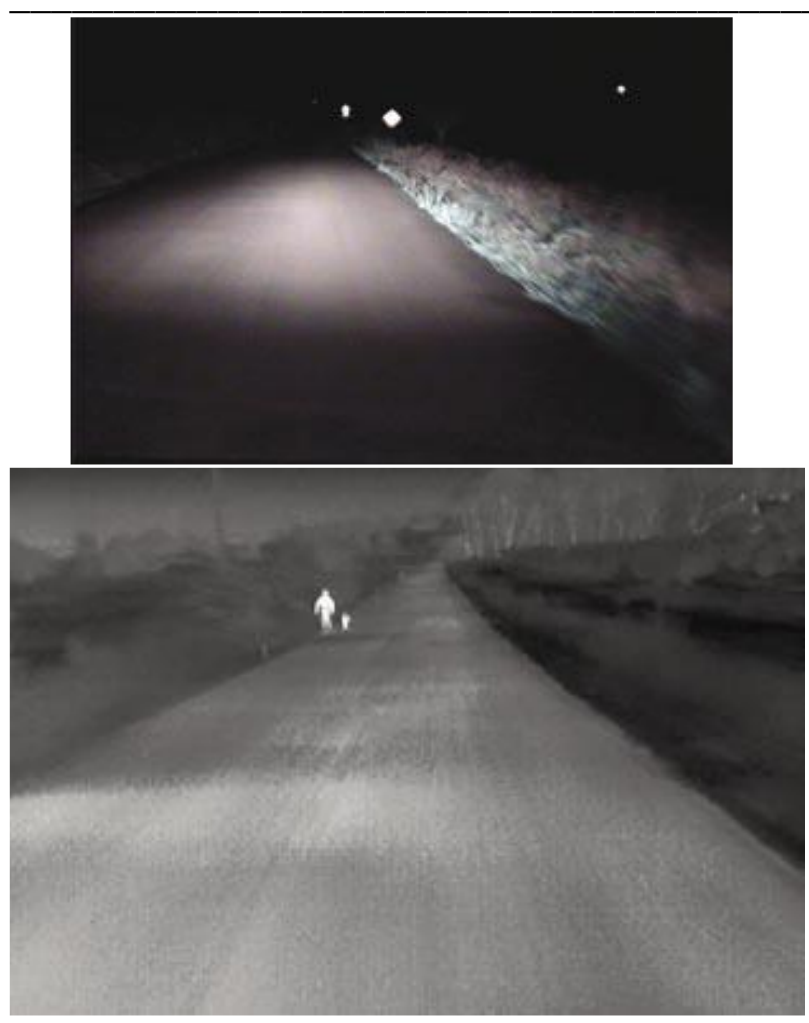

Visual image (in deed no person is identified) Thermal image: Two persons on road

\section{/* A basic PIR sensor probe */}

static int probe_pir(void) \{

static unsigned int lastpir;

unsigned int value = lastpir;

lastpir = (unsigned int $)$ pir_sensor.value $(0)$;

return lastpir - value;

\}

/* A basic activator for sending data over radio */

static void activator_send(void) \{

/* send packet */

rimebuf_copyfrom(PACKET_DATA,

sizeof(PACKET_DATA));

abc_send(\&abc);

\}

/* Print out the report */

static void report(int activator, int probe, int

trigged_percent) \{

printf("Activator \%u Probe \%u: \%u\%\%ln", activator,

probe, trigged_percent);

\}

A complete set of callback functions for a self test of radio triggered PIR sensor.

\section{SCISSOR LIFT EQUIPMENT}

The current lifting mechanism (scissor lift) is based on a planar parallel operated pair of deployable mast created by interlinked Scissor Like Elements (SLEs). The Scissor like elements consist of two metallic rods connected parallel with a revolute joint at their centres. Two SLE's are interlinked by pinning two free ends of one unit with two of the other in such a way that the planarity of the structure is maintained along with the fore-mentioned constraints. The maximum height reached by a scissor lift with $N$

SLE's shall be $N$ times the maximum height reached by a lift with a single SLE. Similarly, the dead weight of a lift with a single SLE shall be multiplied by a factor of $N$. The parallel between the two separate planar lifts is maintained by horizontal connecting rods. The number of SLE's used in each planar lift is the same to maintain the lifting plane at a perpendicular to both the planar lifts. This lifting plane in the current model is occupied by a grab plate which is designed so as to support several types of end-effecters to suit industry-specific requirements (section (2.3.2)). This metal plate is supported by means of revolute pinned joints on one edge and rollers on the other. Similarly, the lift is supported by two revolute joints at one edge on the base and two guided rollers on the other edge

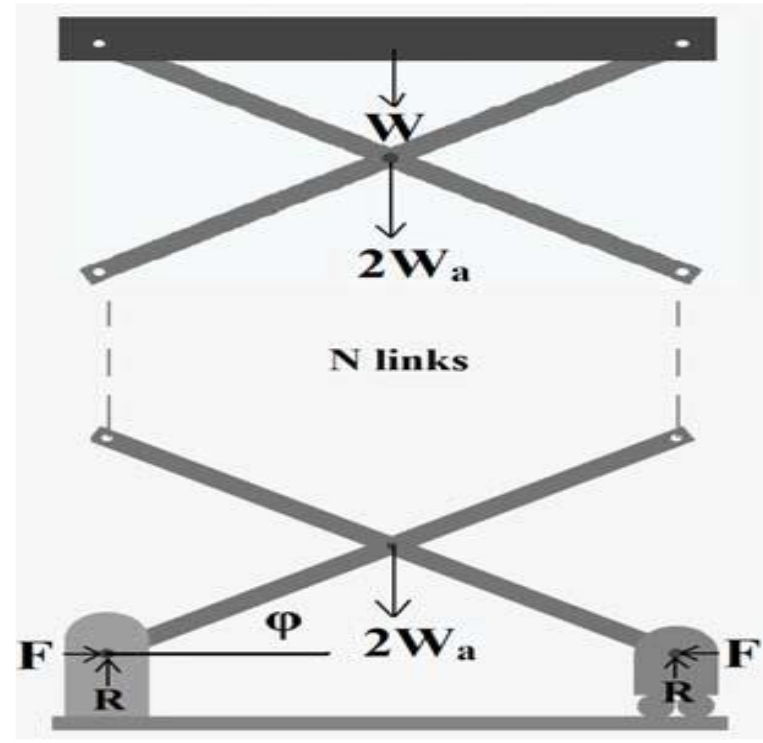

$F=\left(W+\left(N . W_{a}\right)\right) / \tan \varphi$, where $F$ is the force applied by the actuator, $W$ is the net load on the scissor lift inclusive of the weight of the endeffecter plate, $W_{a}$ is 
the weight of each link in the system $N$ is the number of links in the system and $\Phi$ is the angle between the bottom most link of the scissor lift and the base of the robot

\section{Future Scope}

The robot used in this project consists of many sensors, wireless camera and it works efficiently in the places of disaster. The future scope of this project includes the,

- Camera with much more quality

- Reduction in the delay time of robot

\section{CONCLUSION}

Thus the alive human detector using robot has many special features along with many sensors like PIR, Temperature, Intruder, Fire and also has much advantages in the field of rescue and it helps to save many lives as possible than a normal human can do since it has an arm which picks and place those alive persons quickly. This project is considered as very effective and life saving in the field of earthquake, landslides and other natural disasters.

\section{REFERENCES}

[1]. P. B. Tzvi, A. A. Goldenberg, J. W. Zu, "Design,Simulations and Optimization of a Tracked Mobile Robot Manipulator with Hybrid Locomotion and ManipulationCapabilities" Proceedings. of IEEE International Conference on Robotics and automation, pp 2307-2312.2010 (NEAREST IEEE RESEARCH PAPER)

[2] http://www.tinyos.net

[3] http://www.open-zb.net

[4] http://www.robosoft.fr/wifibot.html

[5] http://www.meshcube.org/index_e.html 